\title{
Exosomal miR-34b inhibits proliferation and the epithelial-mesenchymal transition by targeting Notch2 in ovarian cancer
}

\author{
SHENGLIAN LU ${ }^{1 *}$, WUXIA LIU ${ }^{2 *}, \mathrm{HONG} \mathrm{SHI}^{3 *}$ and HAN ZHOU ${ }^{4}$ \\ Departments of ${ }^{1}$ Obstetrics and Gynecology, ${ }^{2}$ Special Clinic and ${ }^{3}$ Ultrasound, Changhai Hospital, Second Military \\ Medical University, Shanghai 200433; ${ }^{4}$ Center for Reproductive Medicine, The Second \\ People's Hospital of Changzhou, Changzhou, Jiangsu 213003, P.R. China
}

Received August 30, 2019; Accepted May 29, 2020

DOI: $10.3892 / \mathrm{ol} .2020 .11837$

\begin{abstract}
Exosomal microRNA (miR) can affect signaling pathways in various physiological and pathological conditions, including ovarian cancer (OC). miR-34b, the first microRNA targeted in a human clinical trial for cancer treatment, exhibited decreased expression in several cancer types. However, the biological function of exosomal miR-34b in OC has not been elucidated. In the present study, using reverse transcription-quantitative PCR, it was reported that exosomal miR-34b is downregulated in OC cells. Exosomal miR-34b reduced cell proliferation and epithelial-mesenchymal transition (EMT) in the OC cell line SKOV3. In addition, it was confirmed that Notch2, which is upregulated in SKOV3 cells, is a target of miR-34b. Moreover, exosomal miR-34b and Notch2 levels were found to be negatively correlated. The present data highlights the importance of exosomal miR-34b-mediated inhibition of cell proliferation and EMT, suggesting that exosomal miR-34b has value as a diagnostic biomarker and a potential molecular target for the treatment of OC.
\end{abstract}

\section{Introduction}

Ovarian cancer (OC) is one of the most fatal malignancies in gynecologic cancer worldwide owing to its frequent detection at an advanced stage $(1,2)$. Based on the statistics from The American Cancer Society in 2013, OC accounts for $4 \%$ of all cancer types diagnosed in women, with an estimated 22,000 new cases and 14,000 deaths per annum in the United

Correspondence to: Dr Han Zhou, Center for Reproductive Medicine, The Second People's Hospital of Changzhou, 29 Xinglong Lane, Changzhou, Jiangsu 213003, P.R. China

E-mail: zrdlylzh@163.com

*Contributed equally

Key words: exosomal miR-34b, ovarian cancer, proliferation, epithelial-mesenchymal transition, Notch2
States alone $(3,4)$. OC has a low 5-year survival rate, and up to $70 \%$ of patients exhibit invasion and metastasis (5). The epithelial-mesenchymal transition (EMT) and collective cell migration to neighboring tissues are key steps in $\mathrm{OC}$ progression and metastasis, although the precise underlying mechanisms are unclear (6).

MicroRNAs (miRNAs/miRs) are small, single-stranded non-coding RNAs that regulate gene expression by binding to specific target mRNA sequences, thereby degrading mRNAs or inhibiting their translation (7). A previous study reported an association between epithelial ovarian cancer (EOC) and aberrant miRNA regulation (8). Various miRNAs are known to be dysregulated in the advanced stages of OC, suggesting that they are involved in malignancy and metastasis (9).

Additional studies have shown that miRNAs can be secreted into the extracellular space, mostly in the form of exosomes, and function in intercellular communication (10-12). Exosomal miRNAs are also important biomarkers of diagnosis and progression of OC (13). For instance, the expression of members of the miR-373 and miR-200 families were significantly upregulated in patients with OC compared with healthy women (14). A previous study reported that exosomal miR-30a-5p may be a potential biomarker for OC (15). miR-34b belongs to the miR-34 family and is directly transactivated by the p53 tumor suppressor (16). In general, miR-34b can promote apoptosis and cell cycle arrest resulting in p53 activation, thereby acting as a mediator of tumor suppression by p53 (17). Several studies have shown that miR-34b is downregulated in $\mathrm{OC}$, but its mechanism of action and precise role in intercellular communication are poorly understood $(18,19)$.

The present study examined the expression and biological functions of exosomal miR-34b and investigated the mechanisms underlying its role in OC.

\section{Materials and methods}

Cell culture. The human ovarian surface epithelium cell line, IOSE-80 and the OC cell lines SKOV3, A2780 and OVCAR3 were purchased from the Cell Bank of Type Culture Collection of the Chinese Academy of Sciences, and cultured in RPMI-1640 medium (Invitrogen; Thermo Fisher Scientific, Inc.) containing 
10\% FBS, 100 units $/ \mathrm{ml}$ penicillin and $100 \mu \mathrm{g} / \mathrm{ml}$ streptomycin in $5 \% \mathrm{CO}_{2}$ at $37^{\circ} \mathrm{C}$.

Exosome purification. Exosomes were isolated from the culture medium by differential centrifugation. Briefly, the conditioned medium was centrifuged at $1,500 \mathrm{x} g$ for $20 \mathrm{~min}$ at $4^{\circ} \mathrm{C}$ to separate the cells, followed by centrifugation at $12,000 \mathrm{x} \mathrm{g}$ for $35 \mathrm{~min}$ at room temperature and filtration through a $0.22-\mu \mathrm{m}$ filter to remove cell debris. Exosomes were pelleted by ultracentrifugation at $120,000 \times \mathrm{g}$ for $90 \mathrm{~min}$ at room temperature, and washed with PBS. Subsequently, the exosomes were pelleted at $120,000 \mathrm{x} \mathrm{g}$ for $70 \mathrm{~min}$ at room temperature and resuspended in $100 \mu \mathrm{l}$ of PBS.

Nanoparticle tracking analysis (NTA). The Nanosight NTA NS300 (Malvern Instruments, Inc.) was used to identify the concentration and size of isolated exosomes by tracking the Brownian motion of particles. The samples were captured for $60 \mathrm{sec}$ at room temperature with manual shutter.

Electron microscopy. For electron microscopy analysis, exosomes isolated from the cell line, IOSE-80 dissolved in PBS were loaded to carbon-coated nickel grids and negatively stained with $2 \%$ methylamine tungstate (Sigma-Aldrich, Inc; Merck $\mathrm{KGaA}$ ) at $37^{\circ} \mathrm{C}$ for $10 \mathrm{~min}$. After drying at room temperature for $20 \mathrm{~min}$, the samples were viewed using a JEM-1230 transmission electron microscope (Nikon Corporation) at $80 \mathrm{kV}$.

$R N A$ preparation and reverse transcription quantitative $(R T-q) P C R$. Total RNA was extracted from cells or using TRIzol $^{\circledR}$ reagent (Takara Biotechnology Co., Ltd). Briefly, $1 \mu \mathrm{g}$ of total RNA was reverse transcribed into cDNA using the Mir-X ${ }^{\mathrm{TM}}$ miRNA FirstStrand Synthesis kit (Takara Biotechnology Co., Ltd.) at $37^{\circ} \mathrm{C}$ for $60 \mathrm{~min}$ and then $85^{\circ} \mathrm{C}$ for 5 sec. qPCR analyses were conducted using the SYBR Prime Script miRNA RT-PCR kit (Takara Biotechnology Co., Ltd), following the manufacturer's procedure. All reagents used for RT-qPCR were obtained from Takara Biotechnology Co., Ltd. The universal miRNA Reverse and U6 primers were also obtained from Takara Biotechnology Co., Ltd. The miR-34b, the universal miRNA Reverse, U6 and mRNA primer sequences are shown in Table I.

Western blot analysis. Proteins were extracted from cells using RIPA lysis buffer (Beyotime Institute of Biotechnology) and the total protein content was measured using the bicinchoninic acid assay (Beyotime Biotechnology, Inc) by Fluoroskan (Thermo Fisher Scientific Inc.). A total of $20 \mu \mathrm{g}$ protein per lane was loaded onto a $12 \%$ SDS-PAGE gel. Proteins were then transferred to polyvinylidene difluoride membranes, and blocked using 5\% BSA at room temperature for $1 \mathrm{~h}$. Then, immunoblotting was performed with primary antibodies against TSG101 (1:2,000; cat. no. ab125011; Abcam), CD63 (1;2000, cat. no. sc-5275; Santa Cruz Biotechnology Inc.), Notch2 (cat. no. 5732S), E-cadherin (cat. no. 14472), $\mathrm{N}$-cadherin (cat. no. 13116), Snail (cat. no. 3879S) and $\beta$-actin (cat. no. 3700S) (1:2,000; all Cell Signaling Technology, Inc.) at $4^{\circ} \mathrm{C}$ overnight. After washing, the membranes were incubated with goat anti-rabbit or anti-mouse antibody
Table I. Primer sequences used in reverse transcription-quantitative PCR.

Gene Primer sequence $\left(5^{\prime} \rightarrow 3^{\prime}\right)$

miR-34b

Forward

CAATCACTAACTCCACTGCCAT

U6

Forward

Universal Reverse

CGCAAGGATGACACGCAAATTCG

Notch2

Forward

Reverse

CCAGTGCAGGGTCCGAGGT

E-cadherin

Forward

GGGACCCTGTCATACCCTCT

Reverse

GAGCCATGCTTACGCTTTCG

$\mathrm{N}$-cadherin

Forward

Reverse

Snail

Forward

Reverse

AGGCTAGAGGGTCACCGCGTC GCTTTGCAGTTCCGACGCCAC

$\beta$-actin

Forward

AGTCAACTGCAACCGTGTGT

AGCGTTCCTGTTCCACTCAT

Reverse

CCTCCC TGTCAGATGAGGAC

CCAGG CTGAGGTATTCCTTG

miR, microRNA.

conjugated to horseradish peroxidase (1:2,000; Cell Signaling Technology) for $1 \mathrm{~h}$ at room temperature. An enhanced chemiluminescence system (Pierce; Thermo Fisher Scientific Inc.) was used to detect the bands. Densitometry was performed using ImageJ v1.8.0 software (National Institutes of Health).

Proliferation assay. Cell Counting Kit (CCK)-8 assays (Dojindo Molecular Technologies, Inc.) were used to evaluate cell viability. Briefly, exponentially growing cells were counted and seeded in 96-well plates at $1 \times 10^{4}$ cells/ well. After 72 h, CCK-8 was added to each well according to the manufacturer's instructions and the plates were incubated for at $37^{\circ} \mathrm{C}$ for $2 \mathrm{~h}$. Then, the absorbance was measured with Fluoroskan (Thermo Fisher Scientific Inc.) at $490 \mathrm{~nm}$.

Wound healing assay. For the wound healing assay, SKOV3 cells were seeded in 6-cm culture dishes. Following serum starvation for $24 \mathrm{~h}$, a straight scratch was created using a $200-\mu 1$ pipette tip and the wound was visualized under a light microscope (magnification, x100). Images of cells were captured under the microscope and the percentage of the scratch healing was calculated.

Transfection. SKOV3 cells were seeded on 6-well plates, and transfected using TurboFect Transfection Reagent (Thermo Fisher Scientific, Inc.) according to the manufacturer's instructions. anti-miR-34b (100 nM), miR-34b mimics $(50 \mathrm{nM})$ and small interfering (si)-Notch2 (100 nM) were 
A

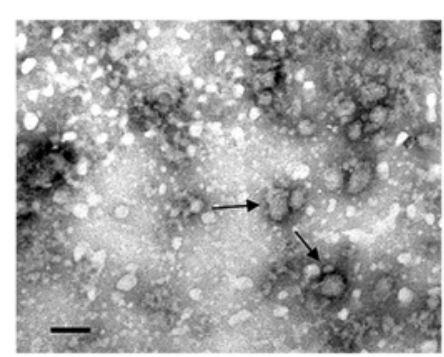

C

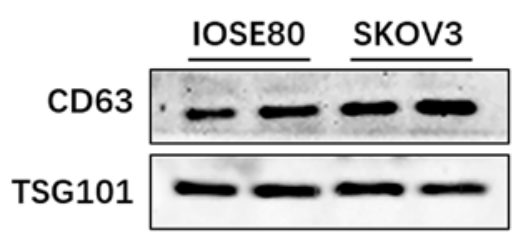

B

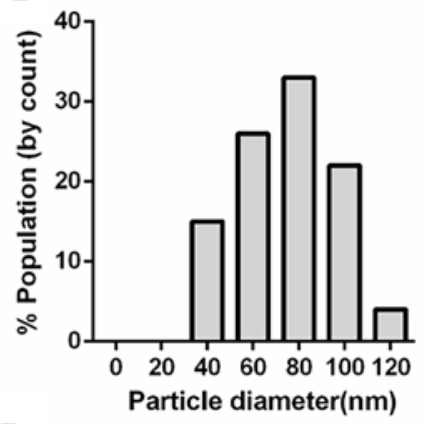

D

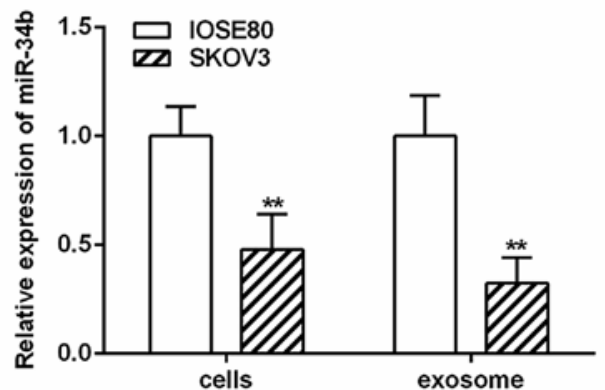

Figure 1. Exosomal miR-34b is downregulated in ovarian cancer. (A) Electron microscopy analysis of exosomes isolated from the SKOV3 conditioned medium. Arrows indicate the exosomes. Scale bar, $100 \mathrm{~nm}$. (B) Size distribution graph of exosomes isolated from the SKOV3 conditioned medium and quantified using nanoparticle tracking analysis. (C) Western blotting of the exosomal markers, CD63 and TSG101, in exosomes isolated from IOSE-80 and SKOV3 conditional medium. (D) Relative expression levels of miR-34b in IOSE-80 and SKOV3 cells and exosomes. ${ }^{* *}$ P<0.01 vs. IOSE-80. miR, microRNA.

used to regulate the expression of miR-34b and Notch2. Scrambled miR-control and silencer negative control 1 siRNA were used as negative controls. Cells were collected $48 \mathrm{~h}$ post-transfection for further experimentation. The sequences used were as follows: miR-34b mimics sense, 5'-AGGCAGUGUAAUUAGCUGAUUGU-3' and antisense, 5'-AAUCAGCUAAUUACACU GCCUUU-3'; anti-miR-34b 5'-ACAAUCAGCUAAUUACACUGCCU-3'; and si-Notch2 sense, 5'-GGAGGUCUCAGUGGAUAUATT-3' and antisense, 5'-UAUAUCCACUGAGACCUCCTT-3'.

Luciferase reporter assay. SKOV3 cells were evaluated using the recombinant pMIR-reporter luciferase vector (Guangzhou RiboBio Co., Ltd.) with a luciferase reporter assay (Promega Corporation). The same transfection methodology was used as described in the previous paragraph. Briefly, cells were transfected with the Notch2 3' untranslated region (UTR) wildtype (WT) and Notch2 3'UTR mutant (Mut) plasmids, together with the negative control RNA and miR-34b mimics using TurboFect Transfection reagent (Thermo Fisher Scientific, Inc.) in 24-well plates. pRL-CMV luciferase plasmids served as the internal control. At $48 \mathrm{~h}$ after transfection, cells were harvested and lysed. Luminescence was determined using the dual-luciferase reporter assay system (Promega Corporation). Firefly luciferase activity was normalized to Renilla luciferase activity.

Prediction of targets of $m i R-34 b$. Three online prediction algorithms PicTar (https://pictar.mdc-berlin.de/), TargetScan (http://www.targetscan.org/vert_71/) and miRDB (http://mirdb.org/) were used to identified targets gene of miR-34b and then the common genes selected from the 3 algorithms were identified as the candidates.
Statistical analysis. All data are presented as mean \pm standard error of the mean from three individual experiments. GraphPad Prism version 7 (GraphPad Software) was used for data analyses. Paired Student's t-test and a one-way analysis of variance (ANOVA) with Tukey's post hoc test were used to assess the differences. Pearson's correlation analysis was used to analyze the correlation between the expression of exosomal miR-34b and Notch 2 mRNA. P $<0.05$ was considered to indicate a statistically significant difference.

\section{Results}

Exosomal miR-34b expression is downregulated in OC cells. Transmission electron microscopy and NanoSight analysis showed that IOSE-80 exosomes had a double-layer membrane and were primarily $40-100 \mathrm{~nm}$ in diameter (Fig. 1A and B). Western blotting further confirmed the presence of exosomes by identifying the well-established markers, CD63 and TSG101 (Fig. 1C). RT-qPCR revealed that miR-34b was highly expressed in the IOSE-80 cell line, and had decreased levels in SKOV3 cells compared with several other OC cell lines (A2780 and OVCAR3) (Fig. S1; ${ }^{*} \mathrm{P}<0.05 ;{ }^{* *} \mathrm{P}<0.01$ ). Therefore, IOSE-80 and SKOV3 cells were selected for further study. The RT-qPCR results showed that miR-34b levels were significantly lower in SKOV3-derived exosomes (SK-Exos) compared with those in the IOSE-80-derived exosomes (IO-Exos), consistent with the cellular expression (Fig. 1D; $\left.{ }^{* *} \mathrm{P}<0.01\right)$.

Exosomal miR-34b attenuates proliferation and EMT in ovarian cancer. RT-qPCR revealed that miR-34b expression was significantly upregulated in SKOV3 co-cultured with 
A

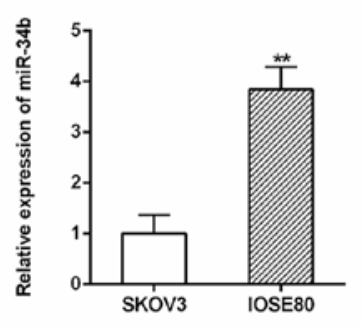

D

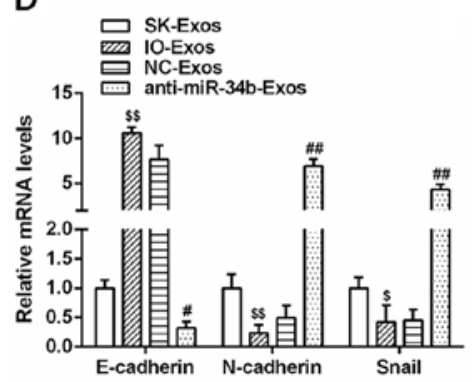

B

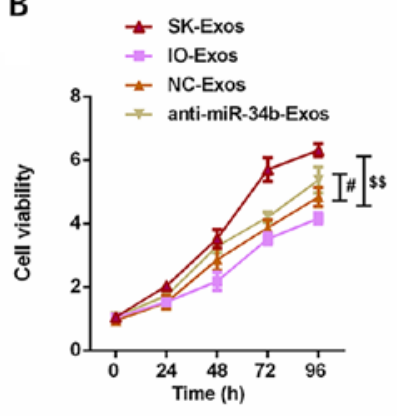

C

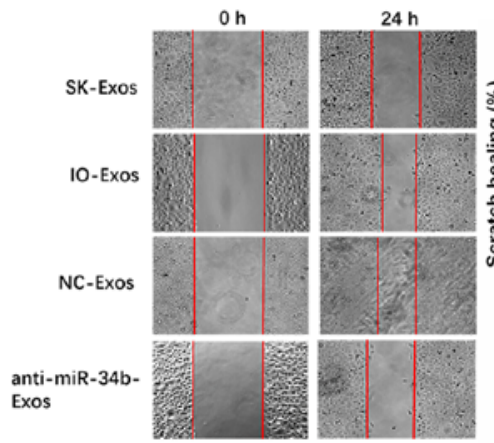

$F$

E
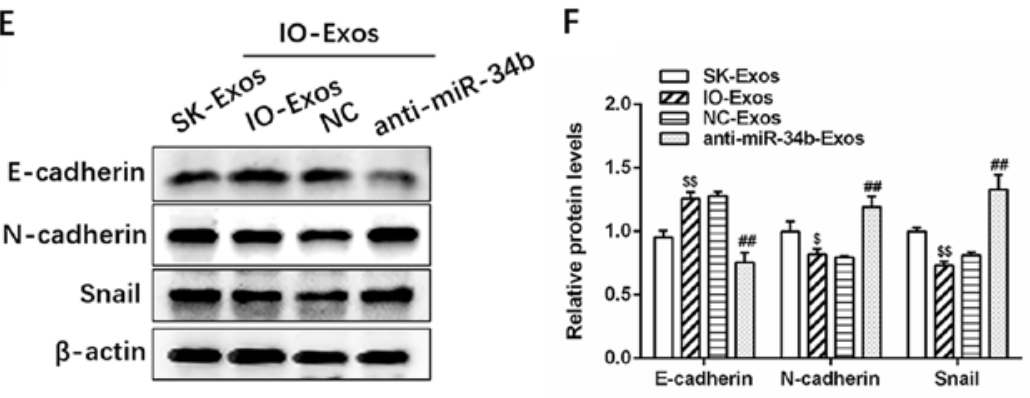

Figure 2. Exosomal miR-34b suppresses cell proliferation and EMT in ovarian cancer cells. (A) Relative expression levels of miR-34b in SKOV3 cells co-cultured with SKOV3 or IOSE-80. (B) Cell viability assays and (C) cell invasion assays in SKOV3 cells cultured with exosomes isolated from SKOV3, IOSE-80, IOSE-80+NC or IOSE-80+anti-miR-34b. (D) Relative expression levels of EMT markers (E-cadherin, N-cadherin and Snail) in SKOV3 cells co-cultured with SKOV3, IOSE-80, IOSE-80+NC or IOSE-80+anti-miR-34b. (E) and (F) Western blotting analysis of EMT markers (E-cadherin, N-cadherin, and Snail) $(20,21)$ in SKOV3 cells co-cultured with SKOV3, IOSE-80, IOSE-80+NC or IOSE-80+anti-miR-34b. ${ }^{* *} \mathrm{P}<0.01$ vs. SKOV3. ${ }^{\$} \mathrm{P}<0.05$, ${ }^{\$ \$} \mathrm{P}<0.01$ and ${ }^{\$ \$} \mathrm{P}<0.001$ vs. SK-Exos, ${ }^{\#} \mathrm{P}<0.05,{ }^{\# \#} \mathrm{P}<0.01$ vs. NC-Exos. EMT, epithelial-mesenchymal transition; NC, negative control; miR, microRNA; SK, SKOV3; IO, IOSE-80; Exos, exosomes.

A

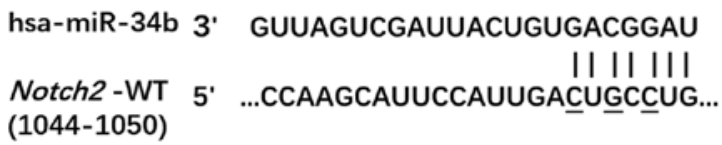

Notch2 -Mut 5' ...CCAAGcauUccauUgatutCaUG...

C

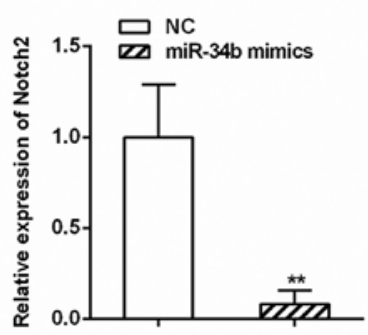

B

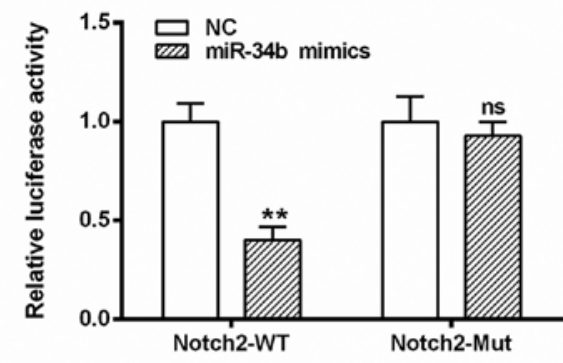

E

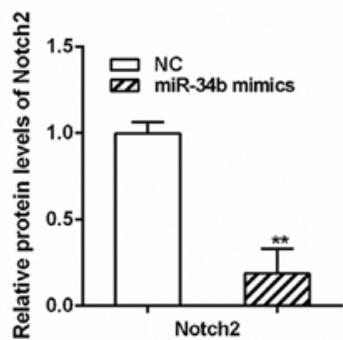

Figure 3. Notch2 is a direct target gene of miR-34b. (A) Predicted miR-34b binding sites in the 3' UTR of Notch2 mRNA. (B) Luciferase activity in cells $48 \mathrm{~h}$ after transfection. (C) Relative expression levels of Notch2 in SKOV3 cells transfected with NC or miR-34b mimics. (D) and (E) Western blotting of Notch2 in SKOV3 cells transfected with NC or miR-34b mimics. ${ }^{* *} \mathrm{P}<0.01$ vs. NC. miR, microRNA; UTR, untranslated region; NC, negative control; ns, not significant.

IOSE-80 cells compared with that of SKOV3 cells alone (Fig. 2A; ${ }^{* *} \mathrm{P}<0.01$ ), suggesting that miR-34b containing exosomes were delivered from IOSE- 80 cells in the upper Transwell chamber to the recipient SKOV3 cells in the lower chamber. RT-qPCR also revealed anti-miR-34b transfection significantly decreased miR-34b expression in exosomes compared with IOSE-80 exosomes (NC-Exos) cells (Fig. S2). The CCK 8 and wound healing assays indicated that IO-Exos markedly attenuated the proliferation and invasion of SKOV3 cells, while exosomes derived from miR-34b-antagonized 
A

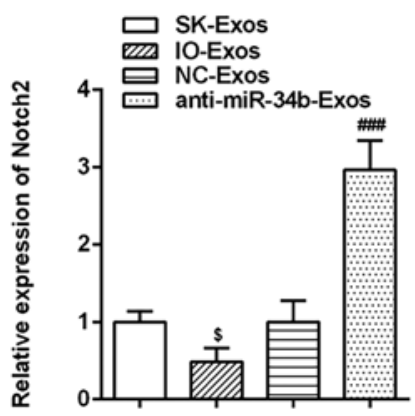

C

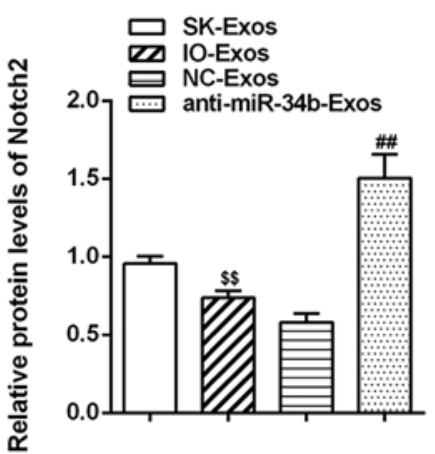

B

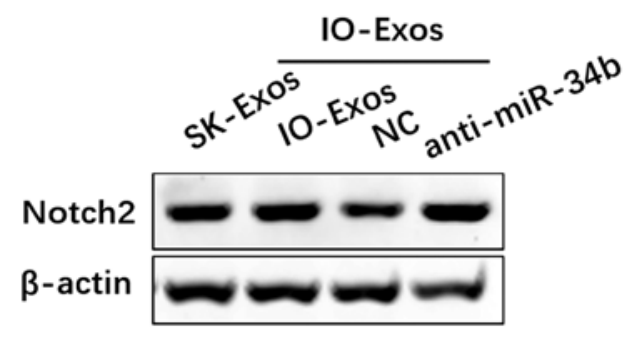

D

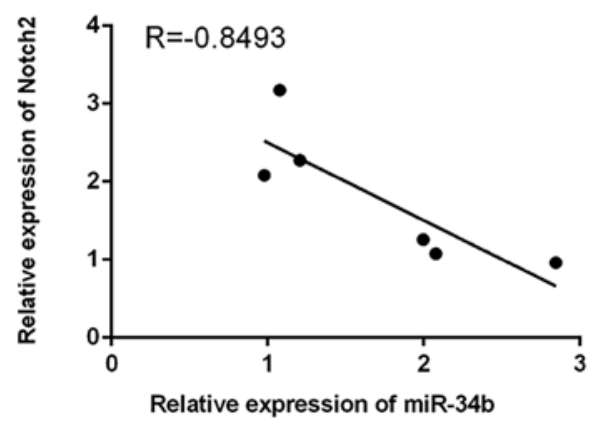

Figure 4. Notch2 is negatively associated with exosomal miR-34b. (A) Relative expression levels of Notch2 in SKOV3 cells cultured with exosomes isolated from SKOV3, IOSE-80, IOSE-80+NC or IOSE-80+anti-miR-34b. (B) and (C) Western blotting of Notch2 in SKOV3 cells co-cultured with SKOV3, IOSE-80, IOSE-80+NC or IOSE-80+anti-miR-34b. (D) Notch2 is negatively correlated with exosomal miR-34b. ${ }^{\$} \mathrm{P}<0.05,{ }^{\$ \$} \mathrm{P}<0.01 \mathrm{vs}$. SK-Exos. ${ }^{\# \#} \mathrm{P}<0.01,{ }^{\# \# \#} \mathrm{P}<0.001$ vs. NC-Exos. miR, microRNA; SK, SKOV3; IO, IOSE-80; Exos, exosomes.

IOSE-80 cells decreased IOSE-80 (IO)-Exos-induced target cell proliferation and invasion (Fig. $2 \mathrm{~B}$ and $\mathrm{C}$; ${ }^{\#} \mathrm{P}<0.05$; ${ }^{\# \#} \mathrm{P}<0.01 ;{ }^{\$ \$} \mathrm{P}<0.01$ and $\left.{ }^{\$ \$} \mathrm{P}<0.001\right)$.

Furthermore, as demonstrated by RT-qPCR and western blotting, IO-Exos was associated with elevated expression of E-cadherin (epithelial marker) (20) and reduced expression of N-cadherin and Snail (mesenchymal markers) (21) at both the mRNA and protein levels in SKOV3 cells (Fig. 2D-F). In contrast, anti-miR-34b-Exos was associated with reduced E-cadherin and increased N-cadherin and Snail expression (Fig. 2D-F; ${ }^{\#} \mathrm{P}<0.05$; ${ }^{\# \#} \mathrm{P}<0.01 ;{ }^{\$} \mathrm{P}<0.05$ and $\left.{ }^{\$ \$} \mathrm{P}<0.01\right)$. In summary, the aforementioned results demonstrated that exosomal miR-34b attenuates the proliferation and EMT in OC cells.

Notch2 is a direct target of $m i R-34 b$. miRNAs are typically involved in the regulation of target genes. Three online prediction algorithms (PicTar, TargetScan and miRDB) identified Notch2 as a potential target gene of miR-34b, with a putative binding site at base pairs 1044-1050 (Fig. 3A). Elevated Notch signaling activity has been reported in previous studies investigating OC $(22,23)$. The dual-luciferase reporter assay showed that luciferase activity for the WT Notch2 3'-UTR was significantly suppressed in cells transfected with miR-34b mimics compared with the control (Fig. $3 \mathrm{~B} ;{ }^{* *} \mathrm{P}<0.01$ ), while no significant change in activity was observed for Mut Notch2 (Fig. 3B; $\left.{ }^{* *} \mathrm{P}<0.01\right)$. Furthermore, Notch 2 mRNA and protein levels were decreased following treatment with miR-34b mimics (Fig. 3C-E; $\left.{ }^{* *} \mathrm{P}<0.01\right)$. Collectively, these results demonstrated that Notch 2 is a direct target of miR-34b.
Notch2 is negatively correlated with exosomal miR-34b. The mRNA and protein levels of Notch2 were lower in cells co-cultured with IOSE-80 compared with those in SKOV3 cells alone (Fig. 4A-C; ${ }^{\# \#} \mathrm{P}<0.01 ;{ }^{\# \# \#} \mathrm{P}<0.001 ;{ }^{\$} \mathrm{P}<0.05$ and ${ }^{\$} \mathrm{P}<0.01$ ). In contrast, Notch 2 mRNA and protein levels were increased in cells treated with anti-miR-34b-Exos (Fig. 4A-C). These results confirmed that Notch 2 is negatively correlated with exosomal miR-34b (r=-0.8493, Fig. 4D).

Notch2 knockdown decreases the effect of exosomal anti-miR-34b on cell proliferation and EMT in OC. The efficiency of Notch2 siRNA transfection was examined in SKOV3 cells (Fig. S3), and then successfully transfected SKOV3 cells were treated with IO-Exos-NC or anti-miR-34b IO-Exos (Fig. 5A). As demonstrated by the CCK-8 and wound healing assays, Notch2 knockdown significantly inhibited the proliferation and invasion induced by exosomal anti-miR-34b (Fig. 5B and $\mathrm{C}$; ${ }^{* *} \mathrm{P}<0.01 ;{ }^{* * *} \mathrm{P}<0.001{ }^{\#} \mathrm{P}<0.05$ and ${ }^{\# \#} \mathrm{P}<0.01$ ). In addition, exosomal anti-miR-34b-induced EMT inhibition was partly decreased by Notch2-knockdown in SKOV3 cells (Fig. 5D-F; " $\mathrm{P}<0.05 ;{ }^{* *} \mathrm{P}<0.01 ;{ }^{*} \mathrm{P}<0.05$ and $\left.{ }^{\# \#} \mathrm{P}<0.01\right)$. Together, these results suggested that exosomal miR-34b mediates cell proliferation and EMT via Notch2.

\section{Discussion}

Despite improvements in surgical approaches, OC still has a high mortality rate, which can be primarily attributed to late-stage diagnosis and a high rate of metastasis (24). Previous 
A

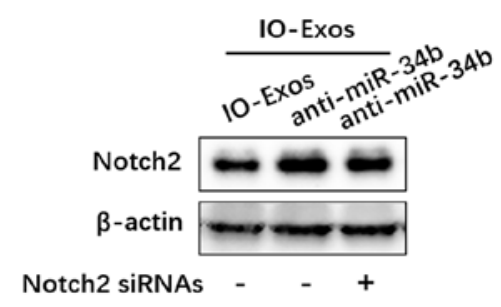

D

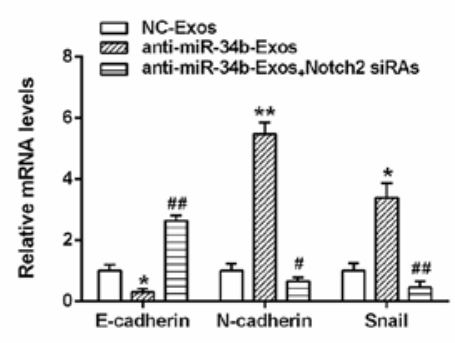

B

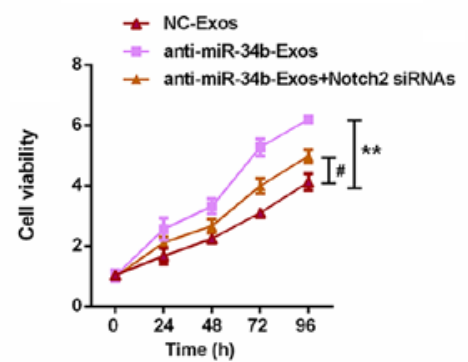

E

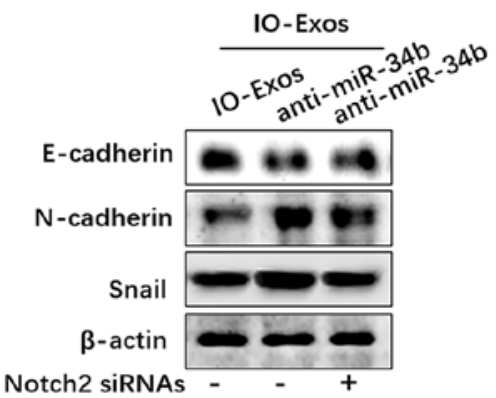

C

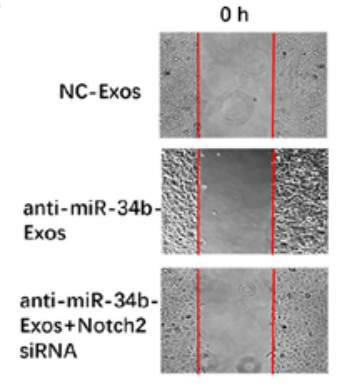

$24 \mathrm{~h}$

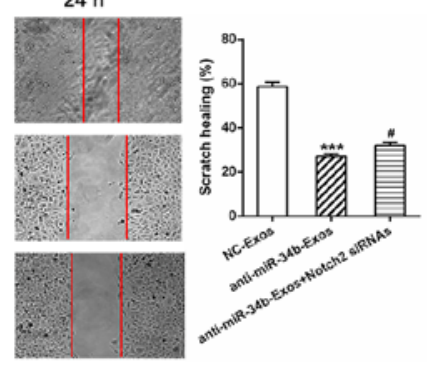

$\mathrm{F}$

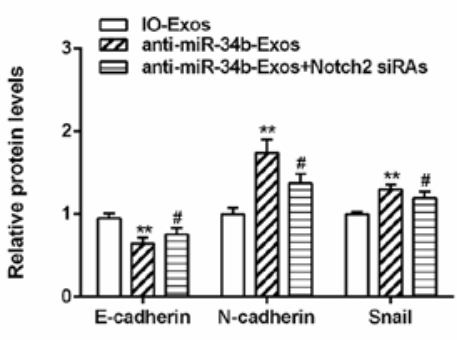

Figure 5. Notch2 knockdown abates the effect of exosomal anti-miR-34b on cell proliferation and the EMT in ovarian cancer cells. (A) Relative expression levels of Notch2 in SKOV3 cells co-cultured with IOSE-80, IOSE-80+NC or IOSE-80+anti-miR-34b. (B) Cell viability and (C) cell invasion assays in SKOV3 cells cultured with exosomes isolated from SKOV-3, IOSE-80, IOSE-80+NC or IOSE-80+anti-miR-34b. (D) Relative expression levels of EMT markers (E-cadherin, N-cadherin and Snail) in SKOV3 cells cultured with exosomes isolated from SKOV3, IOSE-80, IOSE-80+NC or IOSE-80+anti-miR-34b. (E) and (F) western blotting of E-cadherin, N-cadherin and Snail in SKOV3 cells co-cultured with IOSE-80, IOSE-80+NC, or IOSE-80+anti-miR-34b. ${ }^{* *} \mathrm{P}<0.01,{ }^{* * *} \mathrm{P}<0.001$ vs. NC-Exos. ${ }^{~} \mathrm{P}<0.05,{ }^{\# \#} \mathrm{P}<0.01$ vs. anti-miR-34b-Exos. EMT, epithelial-mesenchymal transition; NC, negative control; Exos, exosomes; IO, IOSE-80; SK, SKOV3; si, small interfering.

studies have demonstrated the critical roles of miRNAs in tumorigenesis, progression and metastasis in OC $(25,26)$. Approximately 400 dysregulated miRNAs have been identified in OC, some of which are secreted in exosomes, and are involved in diverse biological functions, including cell proliferation, migration and resistance to paclitaxel and Cisplatin ${ }^{\circledR}(27,28)$. In addition, exosomal miRNAs, including miR-145, miR-940 and Let-7, have been identified as potential diagnostic biomarkers for OC (29-31). Therefore, understanding the roles of dysregulated exosomal miRNAs in OC may help improve diagnosis and treatment. The present study demonstrated that exosomal miR-34b expression is decreased in OC and has an inhibitory effect on cell proliferation and EMT. These results may improve our existing understanding of the fundamental mechanisms underlying the pathogenesis of OC.

miR-34b can act as a tumor suppressor via several mechanisms, including a positive feedback loop between miR-34b and p53 (32). Decreased expression of miR-34b has been detected in various types of cancer, such as colorectal, pancreatic, gastric and ovarian cancer, due to the inactivation of its promoter by CpG methylation (19,33-35). Consequently, miR-34b has become the first miR target to reach the phase 1 clinical trials for solid tumors, such as lung, colon, prostate as well as other disorders, such as cardiac fibrosis, cardiometabolic disease and chronic heart failure $(36,37)$. Moreover, miR-34b has been investigated in body fluids, such as plasma and urine and tissues and has been identified as a biomarker for clear cell renal cell carcinoma $(38,39)$. Several studies have shown that miR-34b expression is lower in OC tissues compared with that in normal ovaries $(16,30)$. However, the functions and underlying mechanisms of action of exosomal miR-34b in OC have not been explored. The present study demonstrated that exosomal miR-34b is highly dysregulated in OC cells. Exosomal miR-34b can suppress cell proliferation and expression of the epithelial adhesion molecule, E-cadherin, and increase the expression of the mesenchymal markers, N-cadherin and Snail, suggesting that miR-34b inhibits EMT in OC $(20,40)$.

Notch2, a member of the Notch family of proteins, is a conserved cell surface receptor that mediates cellular interactions, and is associated with the initiation and development of various types of tumors, such as liver, brain and gastric tumors $(41,42)$. Indeed, Notch signaling has a wide range of functions, and, depending on the cancer type, can function as either a tumor promoter or suppressor, highlighting its complex role in cancer (43). In ovaries, Notch signaling regulates granulosa cell proliferation and coordinates follicular growth; therefore, it is involved in ovarian follicle development (44). In addition, increased Notch2 expression is significantly correlated with poor progression-free survival rate in OC, suggesting that it has prognostic value (45). The present study is the first to report that Notch2 is a direct target gene of miR-34b in OC, to the best of our knowledge. Furthermore, Notch2 was significantly negatively associated with exosomal miR-34b levels, and knockdown of Notch2 abated the decreased cell proliferation and EMT mediated by exosomal anti-miR-34b in OC.

EMT is generally associated with the invasion and migration ability of cells (46). Wound healing and Transwell assays are methods to analyze cell migration. The present study, revealed that exosomal miR-34b attenuates EMT in OC by downregulation of E-cadherin and upregulation of $\mathrm{N}$-cadherin and Snail. However, the Transwell assay was not performed using Matrigel, therefore invasion could not be analyzed, which is a limitation 
of the present study and worthy of further investigation. In addition, the critical role of miR-34b was only explored in OC cell lines, relevant OC animal models should be studied to further confirm the regulatory function of miR-34b in OC.

Overall, the regulatory network of exosomal miR34b and the mechanisms underlying its suppressive effects on cell proliferation and EMT in OC were identified. It was demonstrated that miR-34b acts by directly binding to and regulating Notch2 expression. These findings highlight the important role of exosomal miR-34b in OC, suggesting that it could act as a promising diagnostic and therapeutic target in $\mathrm{OC}$ in the future.

\section{Acknowledgements}

Not applicable.

\section{Funding}

This study was funded by the Changhai Hospital Youth Startup Fund (grant no. 2018QNB004)

\section{Availability of data and materials}

The datasets used and/or analyzed during the present study are available from the corresponding author on reasonable request.

\section{Authors' contributions}

SL and WL designed the study and performed the experiments. HS participated in the experimental design and analysis of data, and drafted and critically revised the manuscript. HZ participated in the experiment design and the subject establishment of this article, in addition he wrote and critically revised the manuscript and gave final approval for the manuscript to be published. All authors read and approved the final manuscript.

\section{Ethics approval and consent to participate}

Not applicable.

\section{Patient consent for publication}

Not applicable.

\section{Competing interests}

The authors declare that they have no competing interests.

\section{References}

1. Karimi-Zarchi M, Mortazavizadeh SMR, Bashardust N, Zakerian N, Zaidabadi M, Yazdian-Anari P and Teimoori S: The clinicopathologic characteristics and 5-year survival rate of epithelial ovarian cancer in Yazd, Iran. Electron Physician 7: 1399-1406, 2015.

2. Siegel R, Ward E, Brawley O and Jemal A: Cancer Statistics, 2011: The impact of eliminating socioeconomic and racia disparities on premature cancer deaths. CA Cancer J Clin 61: 212-236, 2011.

3. Hannibal CG, Cortes R, Engholm G and Kjaer SK: Survival of ovarian cancer patients in Denmark: Excess mortality risk analysis of five-year relative survival in the period 1978-2002. Acta Obstet Gynecol Scand 87: 1353-1360, 2008.
4. Siegel R, Naishadham D and Jemal A: Cancer statistics, 2013. CA Cancer J Clin 63: 11-30, 2013.

5. Ozols RF, Bookman MA, Connolly DC, Daly MB, Godwin AK, Schilder RJ, Xu X and Hamilton TC: Focus on epithelial ovarian cancer. Cancer Cell 5: 19-24, 2004.

6. Chebouti I, Kasimir-Bauer S, Buderath P, Wimberger P, Hauch S, Kimmig R and Kuhlmann JD: EMT-like circulating tumor cells in ovarian cancer patients are enriched by platinum-based chemotherapy. Oncotarget 8: 48820-48831, 2017.

7. Dragomir MP, Knutsen E and Calin GA: SnapShot: unconventional miRNA functions,. Cell 174: 1038-1038.e1, 2018.

8. Reddy KB: MicroRNA (miRNA) in cancer. Cancer Cell Int 15: $38,2015$.

9. De Cecco L, Bagnoli M, Canevari S, Califano D, Perrone F, Pignata S and Mezzanzanica D: miRNA-based signature for predicting epithelial ovarian cancer recurrence. Transl Cancer Res 6 (S1): S232-S234, 2017.

10. Lutgendorf SK, Thaker PH, Arevalo JM, Goodheart MJ, Slavich GM, Sood AK and Cole SW: Biobehavioral modulation of the exosome transcriptome in ovarian carcinoma. Cancer 124: 580-586, 2018.

11. Thomou T, Mori MA, Dreyfuss JM, Konishi M, Sakaguchi M, Wolfrum C, Rao TN, Winnay JN, Garcia-Martin R, Grinspoon SK, et al: Adipose-derived circulating miRNAs regulate gene expression in other tissues. Nature 542: 450-455, 2017.

12. Wei Y, Lai X, Yu S, Chen S, Ma Y, Zhang Y, Li H, Zhu X, Yao L and Zhang J: Exosomal miR-221/222 enhances tamoxifen resistance in recipient ER-positive breast cancer cells. Breast Cancer Res Treat 147: 423-431, 2014.

13. Pan C, Stevic I, Müller V, Ni Q, Oliveira-Ferrer L, Pantel K and Schwarzenbach H: Exosomal microRNAs as tumor markers in epithelial ovarian cancer. Mol Oncol 12: 1935-1948, 2018.

14. Meng X, Müller V, Milde-Langosch K, Trillsch F, Pantel K and Schwarzenbach H: Diagnostic and prognostic relevance of circulating exosomal miR-373, miR-200a, miR-200b and miR-200c in patients with epithelial ovarian cancer. Oncotarget 7: 16923-16935, 2016.

15. Zhou J, Gong G, Tan H, Dai F, Zhu X, Chen Y, Wang J, Liu Y, Chen P, Wu X, et al: Urinary microRNA-30a-5p is a potential biomarker for ovarian serous adenocarcinoma. Oncol Rep 33: 2915-2923, 2015.

16. Corney DC, Hwang CI, Matoso A, Vogt M, Flesken-Nikitin A, Godwin AK, Kamat AA, Sood AK, Ellenson LH, Hermeking H, et al: Frequent downregulation of miR-34 family in human ovarian cancers. Clin Cancer Res 16: 1119-1128, 2010

17. Hosseinpour Z, Salehi Z, Talesh Sasani S and Aminian K: p53 and $\mathrm{miR}-34 \mathrm{~b} / \mathrm{c}$ genetic variation and their impact on ulcerative colitis susceptibility. Br J Biomed Sci 75: 46-49, 2018.

18. Zhang S, Lu Z, Unruh AK, Ivan C, Baggerly KA, Calin GA, Li Z, Bast RC Jr and Le XF: Clinically relevant microRNAs in ovarian cancer. Mol Cancer Res 13: 393-401, 2015.

19. Vogt M, Munding J, Grüner M, Liffers ST, Verdoodt B, Hauk J, Steinstraesser L, Tannapfel A and Hermeking H: Frequent concomitant inactivation of miR-34a and miR-34b/c by $\mathrm{CpG}$ methylation in colorectal, pancreatic, mammary, ovarian, urothelial, and renal cell carcinomas and soft tissue sarcomas. Virchows Arch 458: 313-322, 2011.

20. Zeisberg M and Neilson EG: Biomarkers for epithelial-mesenchymal transitions. J Clin Invest 119: 1429-1437, 2009.

21. Chaw SY, Abdul Majeed A, Dalley AJ, Chan A, Stein S and Farah CS: Epithelial to mesenchymal transition (EMT) biomarkers--E-cadherin, beta-catenin, APC and Vimentin--in oral squamous cell carcinogenesis and transformation. Oral Oncol 48: 997-1006, 2012.

22. Groeneweg JW, Foster R, Growdon WB, Verheijen RH and Rueda BR: Notch signaling in serous ovarian cancer. J Ovarian Res 7: 95, 2014.

23. Zhang CP, Yang JL, Zhang J, Li L, Huang L, Ji SY, Hu ZY, Gao F and Liu YX: Notch signaling is involved in ovarian follicle development by regulating granulosa cell proliferation. Endocrinology 152: 2437-2447, 2011.

24. Yeung TL, Leung CS, Yip KP, Au Yeung CL, Wong ST and Mok SC: Cellular and molecular processes in ovarian cancer metastasis. A Review in the Theme: Cell and molecular processes in cancer metastasis. Am J Physiol Cell Physiol 309: C444-C456, 2015.

25. Srivastava AK, Banerjee A, Cui T, Han C, Cai S, Liu L, Wu D, Cui R, Li Z, Zhang X, et al: Inhibition of miR-328-3p impairs cancer stem cell function and prevents metastasis in ovarian cancer. Cancer Res 79: 2314-2326, 2019. 
26. Zhao W, Han T, Li B, Ma Q, Yang $\mathrm{P}$ and Li H: miR-552 promotes ovarian cancer progression by regulating PTEN pathway. $\mathrm{J}$ Ovarian Res 12: 121, 2019.

27. Deb B, Uddin A and Chakraborty S: miRNAs and ovarian cancer: An overview. J Cell Physiol 233: 3846-3854, 2018.

28. Au Yeung CL, Co NN, Tsuruga T, Yeung TL, Kwan SY, Leung CS, Li Y, Lu ES, Kwan K, Wong KK, et al: Exosomal transfer of stroma-derived miR21 confers paclitaxel resistance in ovarian cancer cells through targeting APAF1. Nat Commun 7: 11150, 2016.

29. Christenson LK: MicroRNA control of ovarian function. Anim Reprod 7: 129-133, 2010.

30. Xie YL, Yang YJ, Tang C, Sheng HJ, Jiang Y, Han K and Ding LJ: Estrogen combined with progesterone decreases cell proliferation and inhibits the expression of Bcl-2 via microRNA let-7a and miR-34b in ovarian cancer cells. Clin Transl Oncol 16: 898-905, 2014.

31. Chen X, Ying X, Wang X, Wu X, Zhu Q and Wang X: Exosomes derived from hypoxic epithelial ovarian cancer deliver microRNA-940 to induce macrophage M2 polarization. Oncol Rep 38: 522-528, 2017.

32. Yamakuchi M and Lowenstein CJ: MiR-34, SIRT1 and p53: The feedback loop. Cell Cycle 8: 712-715, 2009.

33. Hiyoshi Y, Schetter AJ, Okayama H, Inamura K, Anami K, Nguyen GH, Horikawa I, Hawkes JE, Bowman ED, Leung SY, et al: Increased microRNA-34b and -34c predominantly expressed in stromal tissues is associated with poor prognosis in human colon cancer. PLoS One 10: e0124899, 2015.

34. Tsai KW, Wu CW, Hu LY, Li SC, Liao YL, Lai CH, Kao HW, Fang WL, Huang KH, Chan WC, et al: Epigenetic regulation of miR-34b and miR-129 expression in gastric cancer. Int J Cancer 129: 2600-2610, 2011.

35. Liu C, Cheng H, Shi S, Cui X, Yang J, Chen L, Cen P, Cai X, $\mathrm{Lu} \mathrm{Y}, \mathrm{Wu} \mathrm{C}$, et al: MicroRNA-34b inhibits pancreatic cancer metastasis through repressing Smad3. Curr Mol Med 13: 467-478, 2013.

36. Chakraborty C, Sharma AR, Sharma G, Doss CGP and Lee SS: Therapeutic miRNA and siRNA: Moving from bench to clinic as next generation medicine. Mol Ther Nucleic Acids 8: 132-143, 2017.
37. Jung WJ, Koh Y, Kim S, Park H and Yoon SS: Activity of microRNA replacement reagent, MRX34, in multiple myeloma in vivo model. Cancer Res 76: 1081-1081, 2016.

38. Butz H, Nofech-Mozes R, Ding Q, Khella HWZ, Szabó PM, Jewett M, Finelli A, Lee J, Ordon M, Stewart R, et al: Exosomal MicroRNAs Are Diagnostic Biomarkers and Can Mediate Cell-Cell Communication in Renal Cell Carcinoma. Eur Urol Focus 2: 210-218, 2016

39. Ramezani A, Devaney JM, Cohen S, Wing MR, Scott R, Knoblach S, Singhal R, Howard L, Kopp JB and Raj DS: Circulating and urinary microRNA profile in focal segmental glomerulosclerosis: A pilot study. Eur J Clin Invest 45: 394-404, 2015.

40. Wang Y, Shi J, Chai K, Ying X and Zhou BP: The role of snail in EMT and tumorigenesis. Curr Cancer Drug Targets 13: 963-972, 2013.

41. Siebel C and Lendahl U: Notch signaling in development, tissue homeostasis, and disease. Physiol Rev 97: 1235-1294, 2017.

42. Xiu MX and Liu YM: The role of oncogenic Notch2 signaling in cancer: A novel therapeutic target. Am J Cancer Res 9: 837-854, 2019.

43. Koch U and Radtke F: Dual function of notch signaling in cancer: oncogene and tumor suppressor. In: Targeting Notch in Cancer. Miele L and Artavanis-Tsakonas S (eds). Springer, New York, NY, pp55-86, 2018

44. Vanorny DA, Prasasya RD, Chalpe AJ, Kilen SM and Mayo KE Notch signaling regulates ovarian follicle formation and coordinates follicular growth. Mol Endocrinol 28: 499-511, 2014.

45. Chen C, Wang X, Huang S, Wang L, Han L and Yu S: Prognostic roles of Notch receptor mRNA expression in human ovarian cancer. Oncotarget 8: 32731-32740, 2017.

46. Mittal V: Epithelial mesenchymal transition in tumor metastasis. Annu Rev Pathol 13: 395-412, 2018.

(i) (2) This work is licensed under a Creative Commons Attribution-NonCommercial-NoDerivatives $\quad 4.0$ International (CC BY-NC-ND 4.0) License. 